\title{
Real-World Evidence of the Clinical and Economic Impact of Long-Acting Injectable Versus Oral Antipsychotics Among Patients with Schizophrenia in the United States: A Systematic Review and Meta-Analysis
}

\author{
Dee Lin ${ }^{1} \cdot$ Philippe Thompson-Leduc $^{2} \cdot$ Isabelle Ghelerter $^{2} \cdot$ Ha Nguyen $^{3} \cdot$ Marie-Hélène Lafeuille $^{2} \cdot$ \\ Carmela Benson ${ }^{1} \cdot$ Panagiotis Mavros ${ }^{1} \cdot$ Patrick Lefebvre $^{2}$
}

Accepted: 29 March 2021 / Published online: 28 April 2021

(C) The Author(s) 2021, corrected publication 2021

\begin{abstract}
Background Long-acting injectable (LAI) antipsychotics, compared with oral antipsychotics (OA), have been found to significantly improve patient outcomes, including reduced hospitalizations and emergency room (ER) admissions and increased medication adherence among adult patients with schizophrenia. In turn, the clinical benefits achieved may translate into lower economic burden. Real-world evidence of the comparative effectiveness of LAI is needed to understand the potential benefits of LAI outside of the context of clinical trials. This study aimed to provide a comprehensive synthesis of recent published real-world studies comparing healthcare utilization, costs, and adherence between patients with schizophrenia treated with LAI versus OA in the United States.

Methods In this systematic literature review, MEDLINE ${ }^{\circledR}$ was searched for peer-reviewed, real-world studies (i.e., retrospective or pragmatic designs) published in English between January 1, 2010 and February 10, 2020. Comparative studies reporting hospitalizations, ER admissions, healthcare costs, or medication adherence (measured by proportion of days covered $[\mathrm{PDC}]$ ) in adults with schizophrenia treated with LAI versus OA (or pre- vs post-LAI initiation) in the United States were retained. Random effects meta-analyses were conducted among eligible studies to evaluate the association of LAI versus OA use on hospitalizations, ER admissions, healthcare costs, and treatment adherence. A sensitivity analysis among the subset of studies that compared OA with paliperidone palmitate once monthly (PP1M), specifically, was conducted.

Results A total of 1083 articles were identified by the electronic literature search, and two publications were manually added subsequently. Among the 57 publications meeting the inclusion criteria, 25 provided sufficient information for inclusion in the meta-analyses. Compared with patients treated with OA, patients initiated on LAI had lower odds of hospitalization (odds ratio [OR] 0.62, 95\% confidence interval [CI] 0.54-0.71, $n=7$ ), fewer hospitalizations (incidence rate ratio [IRR] [95\% CI] 0.75 [0.65-0.88], $n=9$ ), and fewer ER admissions (IRR [95\% CI] 0.86 [0.77-0.97], $n=6$ ). The initiation of LAI was associated with higher per-patient-per-year (PPPY) pharmacy costs (mean difference [MD] [95\% CI] \$5603 [3799-7407], $n=6$ ), which was offset by lower PPPY medical costs (MD [95\% CI] - \$5404 [ 7745 to -3064$], n=6$ ), resulting in no significant net difference in PPPY total all-cause healthcare costs between patients treated with LAI and those treated with OA (MD [95\% CI] \$327 [ -1565 to 2219], $n=7$ ). Patients initiated on LAI also had higher odds of being adherent to their medication (PDC $\geq 80 \%$; OR [95\% CI] 1.89 [1.52-2.35], $n=9$ ). A sensitivity analysis on a subset of publications evaluating PP1M found results similar to those of the main analysis conducted at the LAI class level.

Conclusions Based on multiple studies with varying sub-types of patient populations with schizophrenia in the United States published in the last decade, this meta-analysis demonstrated that LAI antipsychotics were associated with improved medication adherence and significant clinical benefit such as reduced hospitalizations and ER admissions compared with OA. The lower medical costs offset the higher pharmacy costs, resulting in a non-significant difference in total healthcare costs. Taken together, these findings provide strong evidence on the clinical and economic benefits of LAI compared with OA for the treatment of schizophrenia in the real world.
\end{abstract}

Extended author information available on the last page of the article 


\section{Key Points}

Long-acting injectables were found to significantly reduce the risk and rate of hospitalizations and improve medication adherence for patients with schizophrenia while remaining cost-neutral relative to oral antipsychotics.

Given the inclusion of real-world studies encompassing patient populations with diverse characteristics and healthcare plans, the findings of this study are potentially generalizable to the broader population of patients with schizophrenia in the United States.

\section{Introduction}

Schizophrenia is a debilitating mental disorder, in which distortions in cognition, behavior and emotion severely impair daily functioning and require lifelong treatment [1, 2]. In 2016, roughly 23 million people were affected by schizophrenia worldwide, including 3.5 million people in the United States (US). With an estimated overall cost of $\$ 155.7$ billion in 2013 in the US, schizophrenia places a significant burden on patients, caregivers, payers, and society at large [3-7].

While oral antipsychotics (OA) remain a cornerstone of treatment for schizophrenia, long-acting injectable (LAI) antipsychotics may improve therapeutic continuity and strengthen adherence due to their longer pharmacokinetic half-lives, less frequent dosing frequency, and administration by healthcare providers, compared to daily OA [8]. Current guidelines primarily recommend initiating LAI among patients with a history of poor or uncertain adherence $[9$, 10], although LAI use is also recommended as maintenance therapy or in response to patient preference, while certain guidelines also recommend the use of LAI for first-episode schizophrenia. Furthermore, a recent clinical trial found LAI to be efficacious for the treatment of acute schizophrenia exacerbations [11]. Despite their potential advantages, LAI continue to be underused, most notably in early disease stages, during which use could reduce the risk of poor outcomes associated with medication non-adherence [12-16].

Since a notable advantage of LAI may reside in their lower frequency of administration and associated better adherence, real-world evidence is of particular importance when evaluating the comparative advantage of these medications. Compliance and regular medication intake studied during clinical trials may not translate into benefits of similar magnitude in the real world.

In several real-world studies, patients treated with LAI have shown significantly improved medication adherence and reduced rates of hospitalization and emergency room (ER) admission as compared to those treated with OA [17-30]. Similarly, a randomized trial designed to reflect real-world conditions found LAI to delay time to psychiatric hospitalization as compared to OA [27]. Hospitalizations and ER admissions are often used as a proxy for relapse in the real-world setting and may be of particular interest from a clinical and economic perspective [23, 28-33]. Prior systematic reviews of the literature have focused on the impact of LAI versus OA on hospitalization and have found LAI to be superior to OA in reducing the likelihood and frequency of hospitalization among patients with schizophrenia [18, $32,34]$. However, the most recent publications included in these meta-analyses are from 2016.

To date, multiple real-world studies have assessed healthcare costs in patients with schizophrenia initiated on LAI versus OA [24, 25, 35-39]. In addition to improving adherence, the use of LAI relative to OA may result in considerable medical cost savings due to fewer hospitalizations [24, 25, 35-40]. However, despite the large volume of real-world evidence on the economic burden and adherence patterns of patients with schizophrenia prescribed LAI versus OA in the US [23-26, 35, 37, 40-42], there is, to the best of the authors' knowledge, no recent comprehensive synthesis of these publications.

To fill this knowledge gap, the present systematic review and meta-analysis compared rates of hospitalizations, rates of ER admissions, healthcare costs, and medication adherence among patients with schizophrenia initiated on an LAI versus an OA or before and after initiation of LAI reported in real-world studies in the US.

\section{Methods}

\subsection{Literature Search Strategy}

A systematic literature review was conducted by searching MEDLINE ${ }^{\circledR}$ and MEDLINE In-Process \& Other Citations through the OvidSP interface. The search strategy included a combination of terms to identify schizophrenia, medications indicated for the treatment of schizophrenia, and outcomes of interest. The complete search strategy and screening criteria for the electronic search are presented in Online Resource Table S1 (see the electronic supplementary material). Additional relevant abstracts and posters from 
recent key congresses were added manually to supplement the electronic search.

\subsection{Inclusion Criteria}

Peer-reviewed English language articles published between January 1, 2010 and February 10, 2020 (date of the electronic search) were screened for eligibility. Publications were retained if they met the following inclusion criteria: (1) conducted in a real-world setting (i.e., retrospective administrative claims analyses, retrospective chart review studies, pragmatic trials), (2) included adult patients with schizophrenia, (3) had at least one patient treated in the US, (4) reported at least one outcome of interest, and (5) reported these outcomes comparatively among patients treated with LAI versus OA (two-cohort design) or in the same group of patients before versus after initiation of an LAI (mirrorimage design). Since the clinical endpoints of interest were expected to vary a lot across different countries' healthcare systems, the research was restricted to studies that included patients treated in the US. In addition, only articles published on or after 2010 were retained as treatment patterns prior to 2010 were expected to be meaningfully different, notably through the less prevalent use of LAI.

\subsection{Outcome Measures}

The clinical endpoints of interest were all-cause hospitalizations and ER admissions. In addition, all-cause healthcare costs and medication adherence were reported. All-cause healthcare costs included medical, pharmacy, and total costs. Adherence to the medication of interest (i.e., LAI or OA) was evaluated using the proportion of days covered (PDC).

\subsection{Study Selection and Data Extraction}

Two reviewers (IG and HN) independently assessed each publication for inclusion based on the aforementioned inclusion criteria. Following inclusion, each reviewer independently extracted data, including study design, interventions, sample characteristics, and outcomes, in an Excel-based data extraction grid. All information was taken from the published articles, and authors of the original publications were not contacted. Both extractions were reconciled. Discrepancies in the selection and extraction processes were reviewed and resolved by a third adjudicator (PT-L).

Descriptive statistics including means, medians, and standard deviations (SDs) were extracted for continuous outcomes, while counts and proportions were extracted for binary outcomes. When available in the source publications, odds ratios (ORs) were extracted for binary outcomes, incidence rate ratios (IRRs) were extracted for count outcomes, and mean differences (MDs) were extracted for continuous outcomes.

Because of the heterogeneous study designs and various outcomes of interest included in the scope of this review, a quality assessment exercise was not performed. Rather, a qualitative appraisal of the potential sources of heterogeneity was conducted [43].

\subsection{Meta-Analysis}

Meta-analyses were conducted to evaluate the association of LAI versus OA use on hospitalizations, ER admissions, healthcare costs, and medication adherence. Hospitalizations and ER admissions during the 12-month period following the initiation of an LAI or an OA were analyzed through the number of admissions (12-month pooled IRR) and the odds of having at least one admission (12-month pooled OR). Pharmacy, medical, and total costs per patient per year (PPPY) were analyzed through the cost difference between treatment arms (12-month pooled MD). Adherence was analyzed through the difference in PDC between treatment arms (12-month pooled MD) and the odds of having a PDC $\geq 80 \%$ (12-month pooled OR).

Given the substantial heterogeneity expected between studies, DerSimonian and Laird random effects models were conducted using STATA 16 statistical package (StataCorp LP, College Station, TX, USA). Effect sizes (i.e., ORs, IRRs, and MDs) and confidence intervals (CIs) reported in the source publications were inputted directly in the metaanalyses, when available. For source publications that only reported descriptive statistics, effect sizes and CIs were estimated [44-46].

Among all studies meeting inclusion criteria and reporting outcomes of interest, it happened that multiple publications reported the same outcome from the same data source covering a similar time frame (e.g., cited the same Medicaid or commercial claims insurance database for similar years). Therefore, to avoid the inclusion of overlapping samples, the most representative publication was retained for the metaanalysis based on the following criteria: studies of general schizophrenia populations were prioritized over studies of specific subsets of patients with schizophrenia; studies of LAI overall were prioritized over studies of a single, specific LAI; studies with a cohort design (i.e., LAI vs OA) were prioritized over mirror-image studies (i.e., before vs after LAI initiation).

\subsection{Sensitivity Analysis}

A sensitivity analysis was performed whereby meta-analyses were conducted on studies for which LAI was paliperidone palmitate once monthly (PP1M), exclusively, which was the most common LAI among the publications identified and 
which had previously been found to be used by the majority of patients initiating a second-generation LAI [47].

\section{Results}

\subsection{Study Selection and Characteristics}

A total of 1083 articles were identified by the electronic literature search, and subsequently, two publications presented at relevant conferences at the time that the analysis was conducted were manually added. Partial content was available at the time of the electronic search, and these two studies have since been published as full manuscripts: Zhdanava [48], and Patel [49]). Of these, 57 articles were retained for data extraction (Fig. 1). In total, 25 publications reported at least one outcome of interest for inclusion in meta-analyses. One of these publications presented results on two separate populations (Offord [20], presented outcomes among commercially insured patients and Medicare patients, separately) (Table 1).

Studies were published between 2013 and 2020, and the data included ranged between 2005 and 2018. Study types included 21 retrospective cohort studies $(84 \%)$,
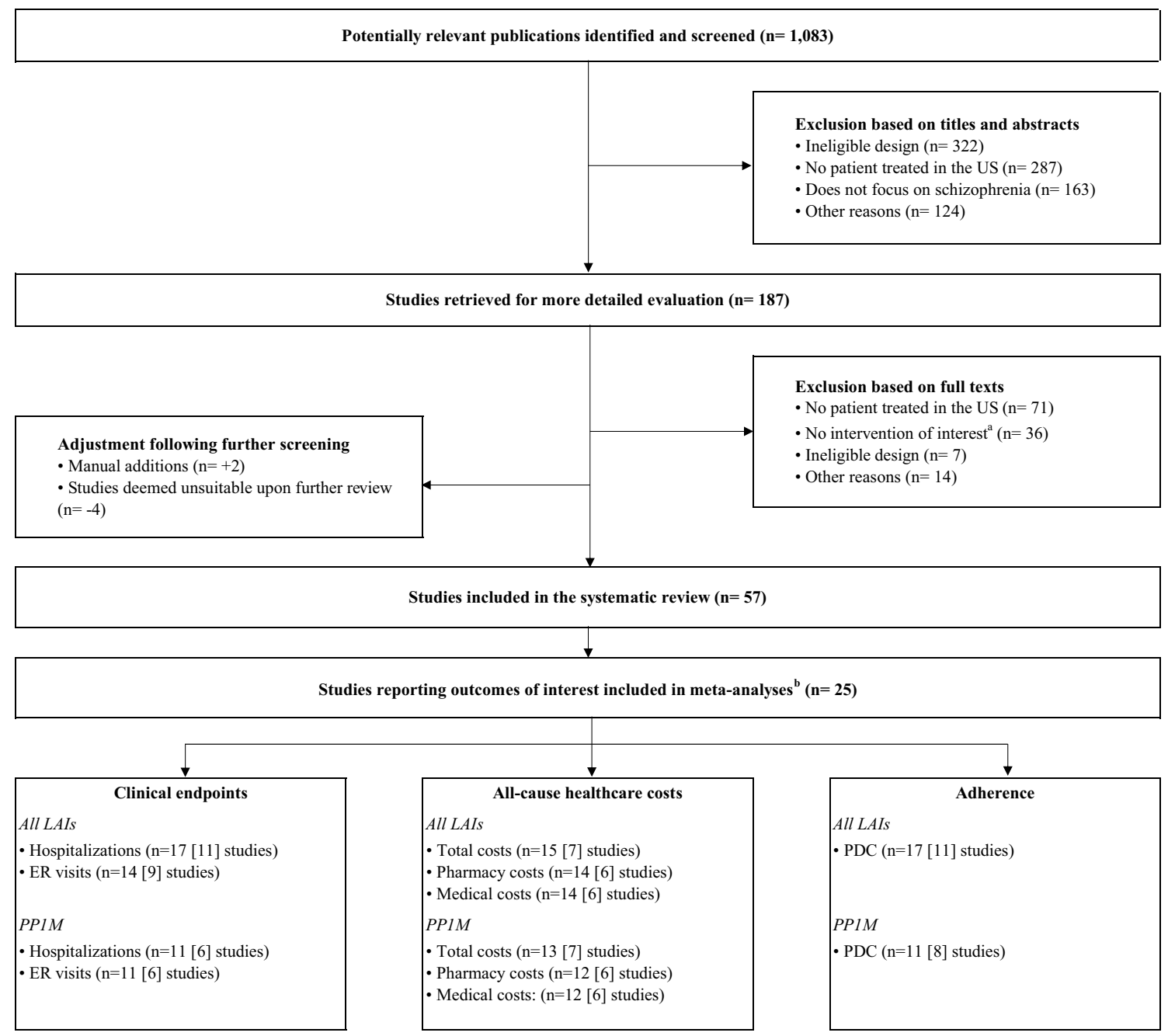

LAI, long-acting injectable; OA, oral antipsychotic; PDC, proportion of days covered; PP1M, paliperidone palmitate once-monthly; US, United States; EA, emergency room

Notes:

${ }^{\mathrm{a}}$ Interventions of interest consisted of comparisons between LAIs and OAs or before and after LAI initiation.

${ }^{\mathrm{b}}$ The counts of studies included for each outcome are presented as the full count and the count after excluding studies with potentially overlapping samples (\# [\#]); e.g., "hospitalizations ( $\mathrm{n}=16$ [10] studies)" indicates that a total of 16 studies reported hospitalization, among which 10 were retained after excluding potentially overlapping samples.

Fig. 1 Study selection flow diagram 
Table 1 Characteristics of studies included in the systematic review

\begin{tabular}{|c|c|c|c|c|c|c|c|c|}
\hline First author, year & Comparison & Population & Years & $n_{\mathrm{LAI}}^{\mathrm{a}}$ & $n_{\mathrm{OA}}{ }^{\mathrm{a}}$ & $\begin{array}{l}\text { Age } \\
\text { Mean } \pm \text { SD }\end{array}$ & $\begin{array}{l}\text { Female } \\
N(\%)\end{array}$ & $\begin{array}{l}\text { Method of } \\
\text { statistical } \\
\text { adjustment }\end{array}$ \\
\hline \multicolumn{9}{|l|}{ Medicaid } \\
\hline $\begin{array}{l}\text { Zhdanava, } 2021 \\
\text { [48] }\end{array}$ & $\begin{array}{l}\text { PP1M (before } \\
\text { and after initia- } \\
\text { tion) }\end{array}$ & Recently relapsed & 2009-2018 & 1725 & 1725 & $39.5 \pm 13.1$ & $743(43.1)$ & Pre-post design \\
\hline Patel, 2021 [49] & PP1M vs. OA & Recently relapsed & 2009-2018 & 208 & 624 & $\begin{array}{l}\text { PP1M: } 38.9 \pm \\
14.3 \\
\text { OA: } 39.9 \pm 14.2\end{array}$ & $\begin{array}{l}\text { PP1M: } 74(35.6) \\
\text { OA: } 216(34.6)\end{array}$ & PSM \\
\hline $\begin{array}{l}\text { Joshi, } 2018(2) \\
\text { [35] }\end{array}$ & PP1M vs. OA & $\begin{array}{l}\text { Comorbid sub- } \\
\text { stance-related } \\
\text { disorders }\end{array}$ & 2009-2015 & 351 & 4869 & $\begin{array}{l}\text { PP1M: } 38.4 \pm \\
11.5 \\
\text { OA: } 41.9 \pm 11.4\end{array}$ & $\begin{array}{l}\text { PP1M: } 101 \\
\quad(28.8) \\
\text { OA: } 2,005(41.2)\end{array}$ & $\begin{array}{c}\text { Multivariable } \\
\text { regressions }\end{array}$ \\
\hline $\begin{array}{l}\text { Lafeuille, } 2018 \\
\text { [65] }\end{array}$ & PP1M vs. OA & $\begin{array}{l}\text { Comorbid diabe- } \\
\text { tes and/or CVD }\end{array}$ & 2009-2015 & 371 & 8296 & $\begin{array}{l}\text { PP1M: } 45.0 \pm \\
10.7 \\
\text { OA: } 47.5 \pm 10.5\end{array}$ & $\begin{array}{l}\text { PP1M: } 167 \\
\quad(45.0) \\
\text { OA: } 4,228(51.0)\end{array}$ & $\begin{array}{c}\text { Multivariable } \\
\text { regressions }\end{array}$ \\
\hline $\begin{array}{l}\text { Manjelievskaia, } \\
2018 \text { [54] }\end{array}$ & PP1M vs. OA & General & 2009-2015 & 949 & 14,649 & $\begin{array}{l}\text { PP1M: } 40.3 \pm \\
\quad 35.7 \\
\text { OA: } 40.0 \pm 9.5\end{array}$ & $\begin{array}{l}\text { PP1M: 3,926 } \\
\quad(51.2) \\
\text { OA: } 4,053(51.1)\end{array}$ & IPTW \\
\hline Shah, 2018 [55] & LAI vs. $\mathrm{OA}^{\mathrm{b}}$ & $\begin{array}{l}\text { Recently diag- } \\
\text { nosed }\end{array}$ & 2010-2015 & 2302 & 2302 & $\begin{array}{l}\text { LAI: } 37.3 \pm \\
13.03 \\
\text { OA: } 37.0 \pm 13.09\end{array}$ & $\begin{array}{l}\text { LAI: } 1,037 \text { (46.6) } \\
\text { OA: } 1,033(44.9)\end{array}$ & PSM \\
\hline $\begin{array}{l}\text { Greene, } 2017 \\
\text { [66] }\end{array}$ & LAI vs. $\mathrm{OA}^{\mathrm{c}}$ & General & 2012-2015 & 2861 & 2777 & $\begin{array}{l}\text { LAI: } 39.9 \pm 13.2 \\
\text { OA: } 42.0 \pm 13.1\end{array}$ & $\begin{array}{l}\text { LAI: } 1,238 \text { (43.3) } \\
\text { OA: } 1,526(55.0)\end{array}$ & $\begin{array}{c}\text { Multivariable } \\
\text { regressions }\end{array}$ \\
\hline Pesa, 2017 [37] & PP1M vs. OA & General & 2008-2014 & 722 & 722 & $\begin{array}{l}\text { PP1M: } 40.4 \pm \\
\text { 12.4 } \\
\text { OA: } 41.2 \pm 12.5\end{array}$ & $\begin{array}{l}\text { PP1M: } 331 \\
\quad(45.8) \\
\text { OA: } 325(45.0)\end{array}$ & PSM \\
\hline $\begin{array}{l}\text { Pilon, } 2017 \text { (1) } \\
\text { [22] }\end{array}$ & LAI vs. OA $^{\mathrm{d}}$ & General & 2009-2015 & 3307 & 21,355 & $\begin{array}{l}\text { LAI: } 41.8 \pm 12.8 \\
\text { OA: } 44.2 \pm 13.5\end{array}$ & $\begin{array}{l}\text { LAI: } 1,340(40.5) \\
\text { OA: } 10,675 \\
\quad(50.0)\end{array}$ & $\begin{array}{c}\text { Multivariable } \\
\text { regressions }\end{array}$ \\
\hline $\begin{array}{l}\text { Pilon, } 2017 \text { (2) } \\
\text { [24] }\end{array}$ & PP1M vs. OA & General & 2008-2015 & 2053 & 22,247 & $\begin{array}{l}\text { PP1M: } 42.9 \pm \\
12.9 \\
\text { OA: } 43.6 \pm 13.4\end{array}$ & $\begin{array}{l}\text { PP1M: } 5,388 \\
\quad(46.4) \\
\text { OA: } 6,293(49.6)\end{array}$ & IPTW \\
\hline $\begin{array}{l}\text { Pilon, } 2017 \text { (4) } \\
\text { [47] }\end{array}$ & LAI vs. $\mathrm{OA}^{\mathrm{e}}$ & General & 2008-2015 & 2209 & 20,478 & $\begin{array}{l}\text { LAI: } 42.2 \pm 12.8 \\
\text { OA: } 44.8 \pm 13.2\end{array}$ & $\begin{array}{l}\text { LAI: } 875(39.6) \\
\text { OA: } 10,006 \\
(48.9)\end{array}$ & $\begin{array}{c}\text { Multivariable } \\
\text { regressions }\end{array}$ \\
\hline Xiao, 2016 ${ }^{f}$ [67] & PP1M vs. OA & $\begin{array}{l}\text { Schizoaffective } \\
\text { disorder }\end{array}$ & 2009-2013 & 876 & 10,778 & $\begin{array}{l}\text { PP1M: } 42.6 \pm \\
31.8 \\
\text { OA: } 43.0 \pm 9.7\end{array}$ & $\begin{array}{l}\text { PP1M: } 2,966 \\
\quad(53.1) \\
\text { OA: } 3,277(54.0)\end{array}$ & Multiple \\
\hline $\begin{array}{l}\text { Kamat, } 2015 \\
\text { [68] }\end{array}$ & $\begin{array}{l}\text { LAI (before and } \\
\text { after initiation) }^{\mathrm{g}}\end{array}$ & General & 2006-2010 & 3094 & 3094 & $38.7 \pm 12.0$ & $1,392(45.0)$ & Pre-post design \\
\hline $\begin{array}{r}\text { Campagna, } \\
2014[52]\end{array}$ & PP1M vs. OA & General & 2008-2011 & 195 & 369 & $\begin{array}{l}\text { PP1M: } 37.9 \pm \\
12.2 \\
\text { OA: } 38.0 \pm 12.4\end{array}$ & $\begin{array}{l}\text { PP1M: } 89 \text { (45.6) } \\
\text { OA: } 205 \text { (55.6) }\end{array}$ & Not described \\
\hline \multicolumn{9}{|l|}{ VHA } \\
\hline $\begin{array}{l}\text { El Khoury, } 2019 \\
\text { [69] }\end{array}$ & $\begin{array}{l}\text { PP1M (before } \\
\text { and after initia- } \\
\text { tion) }\end{array}$ & $\begin{array}{l}\text { Transition from } \\
\text { oral risperi- } \\
\text { done/paliperi- } \\
\text { done }\end{array}$ & 2014-2018 & 319 & 319 & $51.6 \pm 14.2$ & $29(9.1)$ & Pre-post design \\
\hline $\begin{array}{l}\text { Lefebvre, } 2017 \\
\text { [36] }\end{array}$ & PP1M vs. OA & $\begin{array}{l}\text { Comorbid sub- } \\
\text { stance-related } \\
\text { disorders }\end{array}$ & 2010-2015 & 1684 & 5188 & $\begin{array}{l}\text { PP1M: } 52.5 \pm \\
16.7 \\
\text { OA: } 51.7 \pm 9.5\end{array}$ & $\begin{array}{l}\text { PP1M: } 207(6.1) \\
\text { OA: } 213(6.1)\end{array}$ & IPTW \\
\hline $\begin{array}{l}\text { Young-Xu, } 2016 \\
\text { [39] }\end{array}$ & PP1M vs. OA & General & 2009-2014 & 2285 & 8005 & $\begin{array}{l}\text { PP1M: } 53.4 \pm \\
17.2 \\
\text { OA: } 53.0 \pm 9.8\end{array}$ & $\begin{array}{l}\text { PP1M: } 503 \\
\quad(10.0) \\
\text { OA: } 479(9.1)\end{array}$ & IPTW \\
\hline Baser, 2015 [25] & PP1M vs. OA & General & 2007-2012 & 335 & 335 & $\begin{array}{l}\text { PP1M: } 51.3 \pm \\
9.9 \\
\text { OA: } 51.2 \pm 10.3\end{array}$ & $\begin{array}{l}\text { PP1M: } 24(7.0) \\
\text { OA: } 29(9.0)\end{array}$ & PSM \\
\hline
\end{tabular}


Table 1 (continued)

\begin{tabular}{|c|c|c|c|c|c|c|c|c|}
\hline First author, year & Comparison & Population & Years & $n_{\mathrm{LAI}}{ }^{\mathrm{a}}$ & $n_{\mathrm{OA}}{ }^{\mathrm{a}}$ & $\begin{array}{l}\text { Age } \\
\text { Mean } \pm \text { SD }\end{array}$ & $\begin{array}{l}\text { Female } \\
N(\%)\end{array}$ & $\begin{array}{l}\text { Method of } \\
\text { statistical } \\
\text { adjustment }\end{array}$ \\
\hline \multicolumn{9}{|c|}{ Other administrative claims ${ }^{\mathrm{h}}$} \\
\hline $\begin{array}{l}\text { Joshi, } 2018(1) \\
\text { [70] }\end{array}$ & PP1M vs. OA & General & 2009-2015 & 295 & 2296 & $\begin{array}{l}\text { PP1M: } 56.0 \pm \\
28.5 \\
\text { OA: } 55.1 \pm 9.2\end{array}$ & $\begin{array}{l}\text { PP1M: } 661 \\
\quad(60.8) \\
\text { OA: } 655(55.0)\end{array}$ & IPTW \\
\hline Yan, 2018 [71] & LAI vs. $\mathrm{OA}^{\mathrm{i}}$ & General & 2012-2016 & 408 & 3361 & $\begin{array}{l}\text { LAI: } 37.3 \pm 13.4 \\
\text { OA: } 43.6 \pm 15.9\end{array}$ & $\begin{array}{l}\text { LAI: } 172(42.2) \\
\text { OA: } 1,751(52.1)\end{array}$ & $\begin{array}{c}\text { Multivariable } \\
\text { regressions }\end{array}$ \\
\hline $\begin{array}{l}\text { Lafeuille, } 2015 \\
\text { [30] }\end{array}$ & PP1M vs. OA & $\begin{array}{l}\text { Hospitalized at } \\
\text { index }\end{array}$ & 2009-2012 & 374 & 45,251 & $\begin{array}{l}\text { PP1M: } 41.1 \pm \\
14.8 \\
\text { OA: } 45.6 \pm 15.6\end{array}$ & $\begin{array}{l}\text { PP1M: } 120 \\
(32.1) \\
\text { OA: } 17,444 \\
(38.5)\end{array}$ & IPTW \\
\hline $\begin{array}{l}\text { Offord, } 2013 \\
\text { (Commercial) } \\
\text { [20] }\end{array}$ & LAI vs. $\mathrm{OA}^{\mathrm{j}}$ & General & $2005-2010$ & 394 & 2610 & $\begin{array}{l}\text { LAI: } 41.7 \pm 15.5 \\
\text { OA: } 37.1 \pm 15.9\end{array}$ & $\begin{array}{l}\text { LAI: } 190(48.2) \\
\text { OA: } 1,298(49.7)\end{array}$ & $\begin{array}{c}\text { Multivariable } \\
\text { regressions }\end{array}$ \\
\hline $\begin{array}{l}\text { Offord, 2013 } \\
\text { (Medicare) [20] }\end{array}$ & LAI vs. $\mathrm{OA}^{\mathrm{j}}$ & General & 2005-2010 & 147 & 518 & $\begin{array}{l}\text { LAI: } 67.2 \pm 9.8 \\
\text { OA: } 73.2 \pm 10.0\end{array}$ & $\begin{array}{l}\text { LAI: } 88(59.9) \\
\text { OA: } 344(66.4)\end{array}$ & Not described \\
\hline \multicolumn{9}{|l|}{ Other } \\
\hline $\begin{array}{l}\text { Rozin, } 2019 \\
\text { [50] }\end{array}$ & LAI vs. $\mathrm{OA}^{\mathrm{k}}$ & $\begin{array}{l}\text { Recently diag- } \\
\text { nosed }\end{array}$ & 2017 & 10 & 14 & $21.9 \pm 2.5$ & $2(8.3)$ & Not described \\
\hline $\begin{array}{l}\text { Joshi, } 2018(3) \\
\text { [53] }\end{array}$ & LAI vs. $\mathrm{OA}^{1}$ & $\begin{array}{l}\text { Enrollment in } \\
\text { REACH-OUT }\end{array}$ & $2010-2013$ & 599 & 281 & $\begin{array}{l}\text { LAI: } 41.1 \pm 12.4 \\
\text { OA: } 42.1 \pm 13.4\end{array}$ & $\begin{array}{l}\text { LAI: } 161(27.5) \\
\text { OA: } 94(34.2)\end{array}$ & Not described \\
\hline $\begin{array}{l}\text { Anderson, } 2017 \\
\text { [72] }\end{array}$ & PP1M vs. OA & $\begin{array}{l}\text { Enrollment in } \\
\text { REACH-OUT }\end{array}$ & 2010-2013 & 482 & 281 & $\begin{array}{l}\text { PP1M: } 41.1 \pm \\
12.6 \\
\text { OA: } 42.1 \pm 13.4\end{array}$ & $\begin{array}{l}\text { PP1M: } 138 \\
\quad(29.0) \\
\text { OA: } 94(34.2)\end{array}$ & Multiple \\
\hline
\end{tabular}

$C V D$ cardiovascular disease, $I P T W$, inverse probability treatment weighting, $L A I$ long-acting injectable(s), $O A$ oral antipsychotic, $P P 1 M$ paliperidone palmitate once monthly, PSM propensity score matching, REACH-OUT Research and Evaluation of Antipsychotic Treatment in Community Behavioral Health Organizations, Outcomes, $S D$ standard deviation, VHA Veterans Health Administration

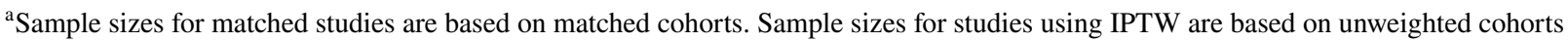

${ }^{\mathrm{b}}$ LAI included aripiprazole, fluphenazine decanoate, haloperidol decanoate, olanzapine pamoate, paliperidone palmitate, and risperidone

${ }^{c}$ LAI included aripiprazole monohydrate, fluphenazine decanoate, haloperidol decanoate, olanzapine pamoate, paliperidone palmitate, and risperidone microspheres

${ }^{\mathrm{d}}$ LAI included aripiprazole, olanzapine, paliperidone palmitate, and risperidone

${ }^{\text {e}} \mathrm{LAI}$ included aripiprazole, fluphenazine decanoate, haloperidol decanoate, olanzapine, paliperidone palmitate, and risperidone

${ }^{\mathrm{f}}$ The study used both PSM and IPTW as method of adjustment. IPTW results are presented here

${ }^{g}$ LAI included fluphenazine, haloperidol, paliperidone, and risperidone

hJoshi, 2018 (1): Humana Research Database; Yan, 2018: Truven Health MarketScan Medicaid, commercial, and supplemental Medicare databases; Lafeuille, 2015: Premier Perspective Comparative Hospital Database; Offord, 2013: MarketScan ${ }^{\circledR}$ Commercial Claims and Encounters and Medicare Supplemental

${ }^{\mathrm{i}}$ LAI included aripiprazole

${ }^{\mathrm{j}} \mathrm{LAI}$ included fluphenazine, haloperidol, and risperidone

${ }^{\mathrm{k}} \mathrm{LAI}$ included aripiprazole and paliperidone palmitate

${ }^{\mathrm{l}} \mathrm{LAI}$ included risperidone and paliperidone palmitate

three pre-post studies (12\%), and one pragmatic trial (4\%). Among retrospective cohort studies $(n=21)$, methods of adjustment to control for confounding included multivariable regressions $(n=7,33 \%)$, inverse probability of treatment weighting ( $n=6,29 \%)$, matching based on propensity scores $(n=4,19 \%)$, and use of multiple techniques ( $n$ $=2,10 \%$ ), and two studies (10\%) did not specify whether methods of adjustment were used.
Data sources included Medicaid (14 studies/25, 56\%), data from the Veterans Health Administration (VHA) (4/25, $16 \%)$, other administrative claims databases $(4 / 25,16 \%)$, and other data types $(3 / 25,12 \%)$.

Sample size varied widely, ranging between 24 [50] and 45,625 [30]. The proportion of females in the publications was noticeably lower (10\% or less) in studies using data from the VHA and in one chart review study on cannabis users [50]. Patients' mean ages among studies using Medicaid data 
were in the late-thirties and forties, and in the early fifties among studies using VHA data.

Additional details on study designs and samples are available in Online Resource Table S2 and Online Resource Table S3 (see the electronic supplementary material).

\subsection{Clinical Endpoints}

Ten studies reported the likelihood of being hospitalized 12 months following initiation of an LAI compared with an OA (Online Resource Table S4A; see the electronic supplementary material). Among the seven studies included in the meta-analysis, patients initiated on an LAI had lower odds of being hospitalized compared with an OA (OR [95\% CI] 0.62 [0.54-0.71], $n=7$ ).

Fifteen studies reported on rates of hospitalization (Online Resource Table S4B). Among the eight studies that were included in the meta-analysis (one of which reported outcomes on two separate samples of patients [20]), patients initiated on an LAI had 25\% fewer all-cause hospitalizations (IRR [95\% CI] 0.75 [0.65-0.88], $n=9$; Fig. 2).

Seven studies reported the likelihood of being admitted to the ER within 12 months of the initiation of an LAI compared with an OA (Online Resource Table S4C). Among the six studies that were included in the meta-analysis, patients initiated on an LAI had lower odds of being admitted to the ER, although this did not reach statistical significance (OR [95\% CI] 0.79 [0.61-1.03], $n=6$ ).

Thirteen studies reported the rate of ER visits following initiation of an LAI compared with an OA (Online Resource Table S4D). Among the six studies that were included in the meta-analysis, patients initiated on an LAI had 14\% fewer all-cause ER admissions (IRR [95\% CI] 0.86 [0.77-0.97], $n=6$; Fig. 3).

\subsection{Healthcare Costs}

Fifteen studies reported total costs following the initiation of an LAI compared with an OA (Online Resource Table S4E; see the electronic supplementary material). Among the seven studies included in the meta-analysis, there was no significant difference in total all-cause healthcare costs PPPY between patients initiated on an LAI versus an OA (MD [95\% CI] \$327 [ 1565 to 2219], $n=7$; Fig. 4). Of these seven studies, six reported pharmacy and medical costs, separately. While the initiation of an LAI was associated with higher PPPY pharmacy costs (MD [95\% CI] \$5603 [3799-7407], $n=6$, Online Resource Table S4F), this was offset by lower PPPY medical costs (MD [95\% CI] - \$5404 [ -7745 to -3064 ], $n=6$, Online Resource Table S4G).

\subsection{Treatment Adherence}

Fourteen studies reported mean PDC among patients initiated on an LAI compared with those initiated on an OA (Online Resource Table S4H; see the electronic supplementary material). Among the nine studies that were included in the meta-analysis, patients initiated on an LAI had a mean PDC nine percentage points higher (MD $[95 \% \mathrm{CI}]$ $9 \%$ [2-15], $n=9)$.

Sixteen studies reported the likelihood of being adherent to an LAI compared with an OA (Online Resource Table S4I). Among the nine studies that were included in the meta-analysis, patients initiated on an LAI were $89 \%$ more likely to be adherent to their medication (OR [95\%

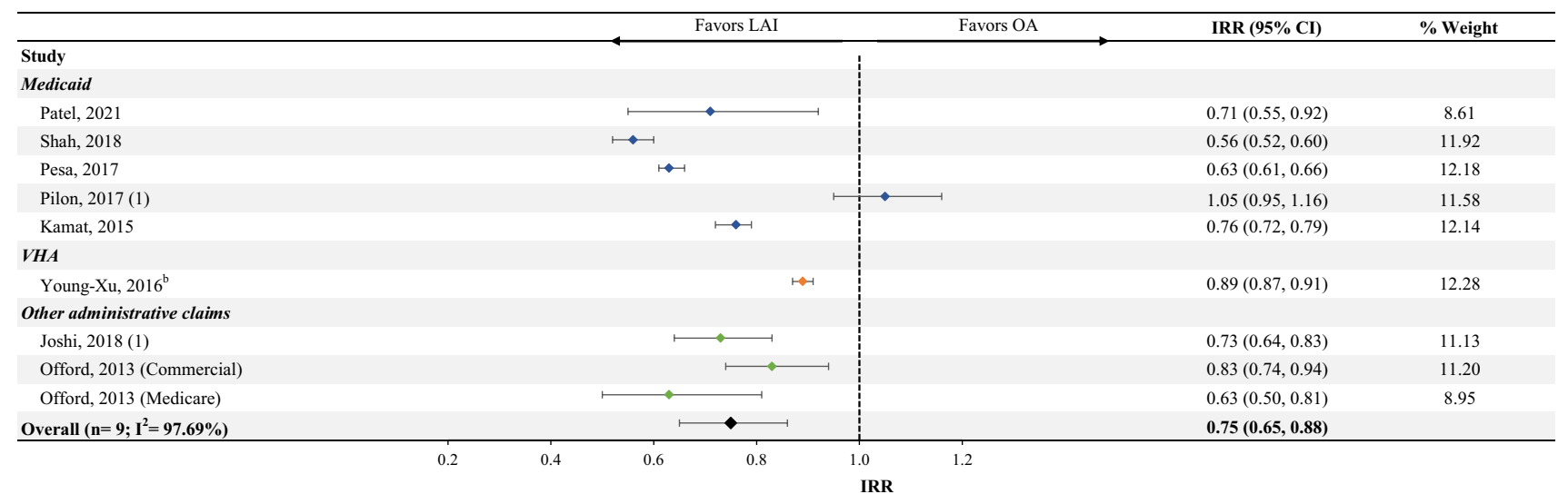

CI: confidence interval; IRR: incidence rate ratio; LAI: long-acting injectable; OA: oral antipsychotic; VHA: Veterans Health Administration

Notes:

${ }^{a}$ This forest plot presents the impact of LAIs on the number of 12-month all-cause hospitalizations among real-world studies using a random effects model of IRRs.

${ }^{\mathrm{b}}$ Patients were required to have $\geq 6$ months of follow-up; outcomes were annualized for patients with $<12$ months of follow-up.

Fig. 2 Number of annual all-cause hospitalizations ${ }^{\mathrm{a}}$ 


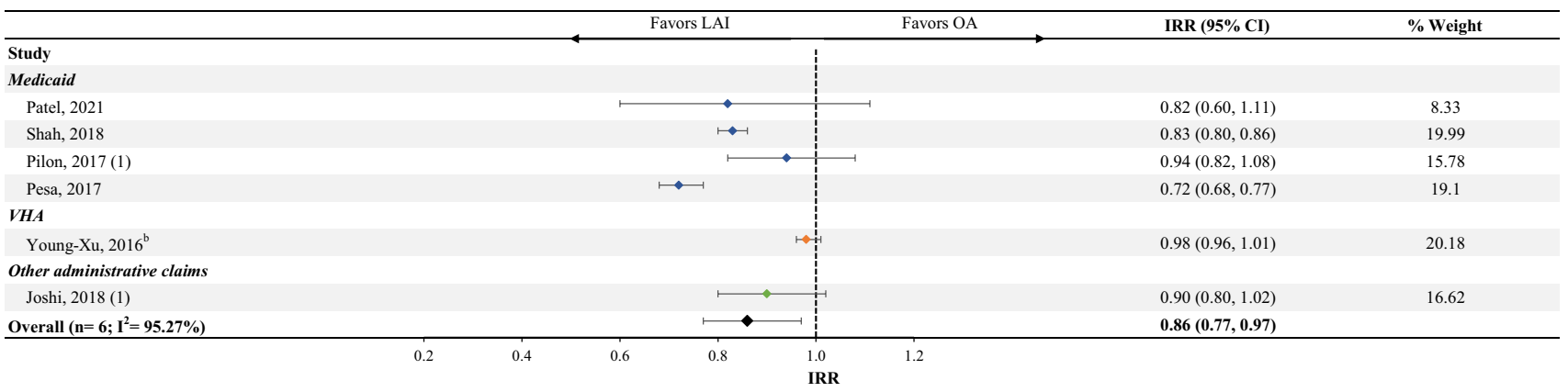

CI: confidence interval; ER: emergency room; IRR: incidence rate ratio; LAI: long-acting injectable; OA: oral antipsychotic; VHA: Veterans Health Administration

Notes:

${ }^{\mathrm{a}}$ This forest plot presents the impact of LAIs on the number of 12-month all-cause ER visits among real-world studies using a random effects model of IRRs.

${ }^{\mathrm{b}}$ Patients were required to have $\geq 6$ months of follow-up; outcomes were annualized for patients with $<12$ months of follow-up.

Fig. 3 Number of annual all-cause ER visits ${ }^{\mathrm{a}}$

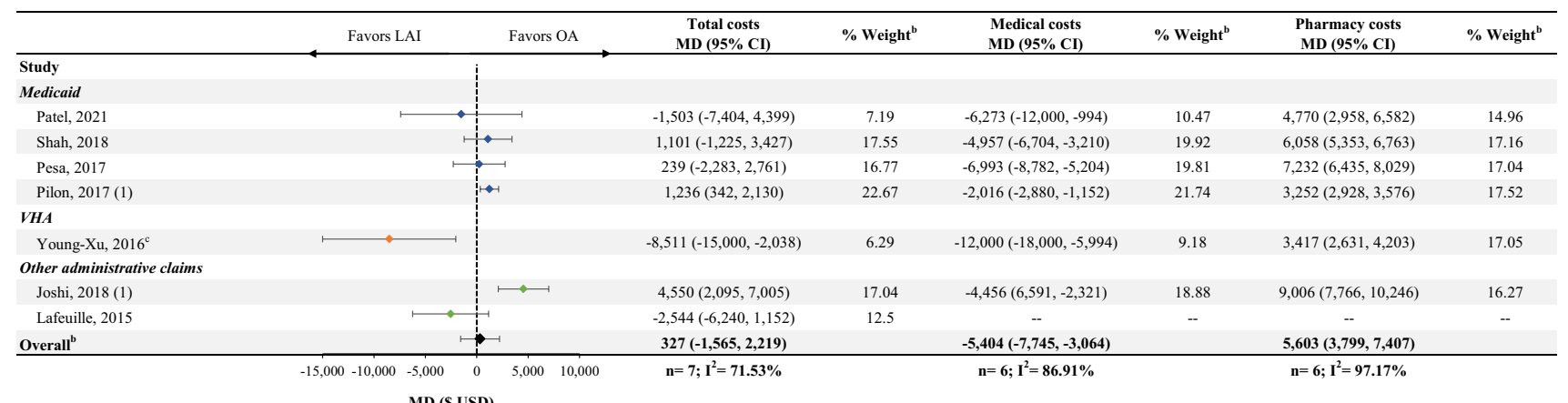

CI: confidence interval; LAI: long-acting injectable; MD: mean difference; OA: oral antipsychotic; USD: United States Dollar:

VHA: Veterans Health Administration

Notes:

a This forest plot presents the impact of LAIs on annual all-cause total healthcare costs among real-world studies using a random effects model of MDs.

${ }^{\mathrm{b}}$ Given that different weights are assigned when combining each outcome and that pharmacy and medical costs were not available for all studies in which total costs were reported, it is expected that the sum of the pooled estimates of the mean differences in medical and pharmacy costs does not equal the pooled estimate of the mean difference in total costs.

${ }^{\mathrm{c}}$ Patients were required to have $\geq 6$ months of follow-up and outcomes were annualized for patients with $<12$ months of follow-up.

Fig. 4 Annual all-cause healthcare $\cos ^{\mathrm{a}}$

CI] 1.89 [1.52-2.35], $n=9$ ) compared with those initiated on an OA (Fig. 5).

\subsection{Sensitivity Analysis}

Articles comparing PP1M with OA, which included 16 publications out of the 25 articles retained, also found similar associations to those of the main analysis across all outcomes. Patients initiated on PP1M had fewer all-cause hospitalizations (IRR [95\% CI] 0.74 [0.62-0.89], $n=6$, Online Resource Fig. S1, see the electronic supplementary material) and ER admissions (IRR [95\% CI] 0.82 [0.69-0.98], $n=6$, Online Resource Fig. S2) compared with patients initiated on an OA. Similarly to the main analysis, the difference in PPPY total healthcare costs between patients treated with PP1M and those treated with an OA was not significant (MD [95\% CI] $\$ 107$ [ -2268 to 2482], $n=7$, Online Resource Fig. S3).
Treatment with PP1M was also associated with greater likelihood of medication adherence as compared to OA (OR [95\% CI] 1.93 [1.54-2.42], $n=8$, Online Resource Figure S4).

\section{Discussion}

This study provides a comprehensive synthesis of the literature with regards to clinical endpoints, healthcare costs, and medication adherence among patients with schizophrenia treated with LAI versus those treated with OA in the US. In this meta-analysis, treatment with LAI was associated with reduced risk and rate of hospitalizations and ER admissions, as well as increased medication adherence, relative to OA. These improved patient outcomes were achieved at no additional total cost to the healthcare system. 


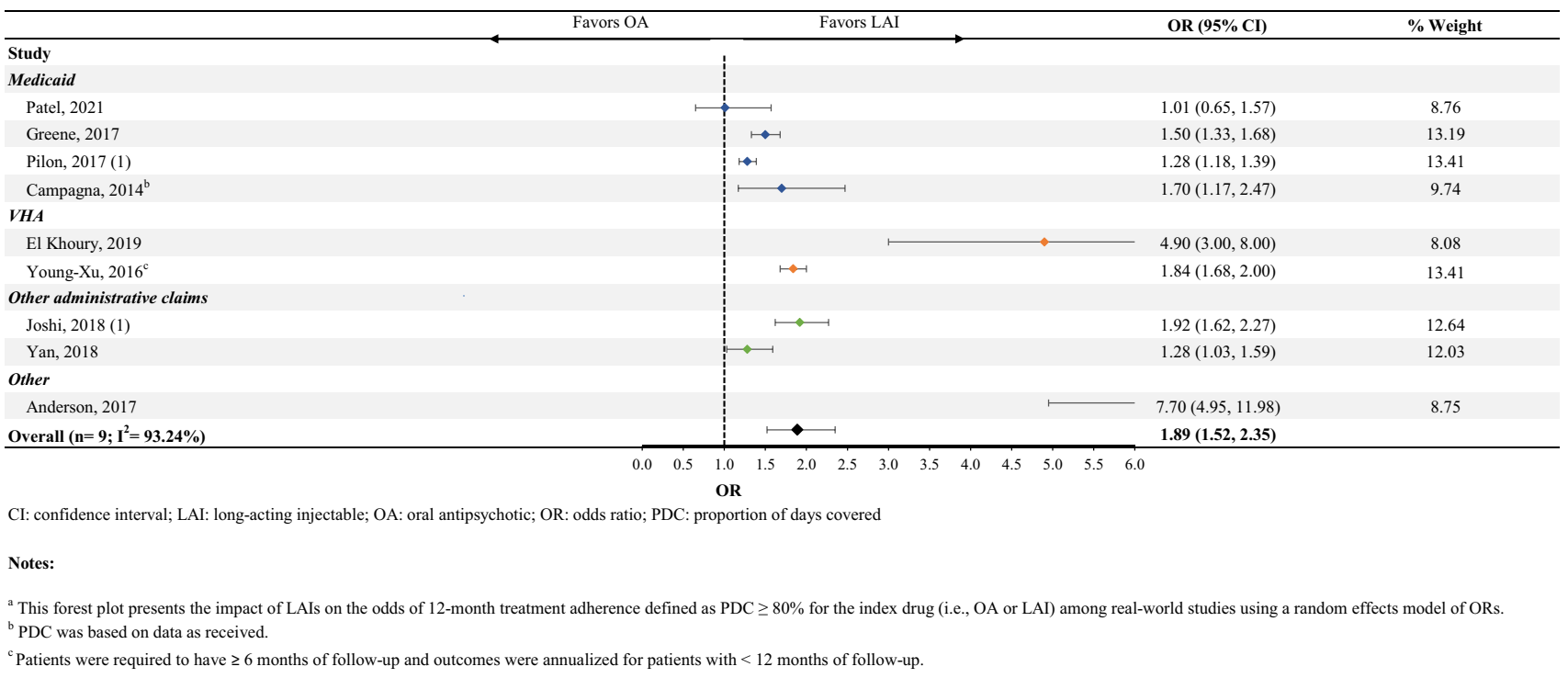

Fig. 5 Treatment adherence $(\mathrm{PDC} \geq 80 \%)^{\mathrm{a}}$

The current literature review focused on real-world studies, allowing for a better real-world representation of hospitalizations, ER admissions, cost outcomes, and adherence patterns of patients.

The publications that were identified through the literature review reported on data from different populations in the US. Notably, they used various databases (e.g., Medicaid, VHA, commercial insurance) that included different healthcare plans with varying cost structures and comprised patients with diverse demographic and clinical characteristics. These comprehensive data sources are a useful way to obtain a patient's entire antipsychotic profile. Given the varying tolerability profiles of different antipsychotics and potential polypharmacy, considering all sources of antipsychotic prescribing (including those outside of primary care) is critical [51].

In addition, diverse adjustment methods were used to control between the LAI and OA cohorts of each study, including pre-post designs, propensity score matching, inverse probability of treatment weighting, and multivariable regressions. Since the scope of the search was relatively narrow (i.e., common pathology, recent timeframe, unique country), the heterogeneity observed across the studies was likely a result of sampling rather than fundamental differences in study objectives. The presence of statistical heterogeneity between the studies was confirmed through the $I^{2}$ statistic and further motivated the need to use random effects models.

While a uniform risk of bias assessment was not conducted for this review, the main study design characteristics of studies included in this meta-analysis were reviewed qualitatively to assess the comparability of studies. Most included studies used a study design that adjusted for differences between study cohorts, which limits the risk of bias due to confounding. Three studies did not report a method of multivariable adjustment, including two retrospective cohort studies [50, 52] and one pragmatic trial [53]. These studies reported on the likelihood of being hospitalized [50, 53], the likelihood of having an ER visit [53], and adherence [52]. In all cases, the studies' point estimates fell within the range of the other studies reporting on these outcomes, and therefore it is unlikely that descriptive studies that did not adjust for confounding materially impacted this review's conclusions.

All publications were reviewed to ensure that a patient who could be included in more than one publication (e.g., same Medicaid sample for the same years) was only included once in the meta-analyses. Publications that were excluded because of potentially overlapping samples reported point estimates that fell within range of other studies reporting on these outcomes, with a few exceptions. Notably, Zhdanava [48] reported the lowest odds of hospitalizations and ER visits among LAI users of any study, but was excluded from the meta-analysis as some patients could overlap with Patel [49] and because it used a pre-post design. Baser [25] reported the lowest IRR of ER visits as well as the largest decrease in medical costs among LAI users, but was excluded as it potentially overlapped with Young-Xu [39] and had a smaller sample size. Manjelievskaia [54] reported the lowest (but still positive) increase in pharmacy costs associated with LAI, but was excluded as it potentially overlapped with Shah [55]. In all cases, the studies' findings were directionally similar to the meta-analysis, and therefore, it is not expected that their inclusion would have changed the conclusions of this review. 
All-cause hospitalizations and ER admissions were selected as outcomes of interest as they are typically considered as proxies for episodes of schizophrenia relapse, which in turn are associated with impaired functioning, reduced quality of life, and potential harm to oneself or others [3, $31,33,56]$. The reduced risk/rate of hospitalization in the present review is consistent with the results of previous literature reviews and meta-analyses based on real-world data $[18,32,34]$. When compared to OA, treatment with LAI was associated with a $21 \%$ greater reduction in hospitalization rates in a meta-regression of both interventional and non-interventional studies [32]. Similarly, treatment with LAI prevented hospitalization and reduced the number of hospitalizations in a meta-analysis of 25 mirror-image studies [18]. In a more recent meta-analysis focused exclusively on non-interventional cohort studies, Kishimoto et al. [34] found that LAI were superior to OA in decreasing hospitalization rate and lowering the risk of hospitalization among patients with schizophrenia in a real-world setting.

Along the same lines, several studies have evaluated schizophrenia-related hospitalization or ER admissions as a proxy for schizophrenia relapses in the real-world setting $[23,28-30]$. Consistent with the current meta-analyses that have focused on all-cause hospitalizations and ER admissions in general, these studies have shown that patients with schizophrenia who are treated with LAI have significantly lower rates of relapse compared to those treated with OA [23, 28-30].

Furthermore, the present review found that the total allcause healthcare costs of LAI and OA were similar, with the medical costs savings associated with LAI offsetting the higher costs of drug acquisition relative to OA. The studies included were based on data spanning 2008 until 2018, across which the consistent result of cost offset suggests generalizability of this finding across time. In addition, these findings are in agreement with observations made in a 2018 review of the clinical and economic burden of commercially insured patients with schizophrenia in the US [55], in which two studies reported that the initiation of LAI was associated with reduced hospitalizations and inpatient costs (compared to pre-LAI initiation and relative to patients initiated on OA), and one study reported that healthcare resource use and costs declined significantly at 6 months after (vs 6 months before) initiating LAI [20, 57, 58]. The offset of higher pharmacy costs by lower medical costs is noteworthy, as stakeholders may be incentivized to prioritize one mode of treatment over the other in cases where pharmacy and medical costs are the responsibility of two different stakeholders.

For this outcome, it is important to consider whether studies that reported on costs were different from those that did not. Among the nine studies reporting on the rate of hospitalization and the seven studies reporting on total costs, six studies overlapped. Studies that did not overlap were not meaningfully different, with the exception of Offord "Medicare" [20], in which patients were older than patients in the other studies, and healthcare costs could differ for such a sample.

In the present review, patients initiated on LAI were significantly more likely to be adherent to treatment than those initiated on OA. According to prior estimates, at least 50\% of patients with schizophrenia initiated on antipsychotics are not adherent to their medication, which poses a major challenge in clinical practice $[59,60]$. The present results suggest that treatment with LAI may serve as an effective strategy for improving adherence and therapeutic continuity in the real-world setting. Given the association between poor medication adherence and disease relapse, the improved adherence associated with LAI is likely a contributing factor to the lower rates of hospitalizations and the associated costs of care [61, 62].

\subsection{Limitations}

The results of the present study should be interpreted in the context of certain limitations. First, this review identified studies through MEDLINE ${ }^{\circledR}$ and MEDLINE In-Process, a broad medical literature database, but did not include other databases, unpublished articles, or articles published in languages other than English. While it is expected that MEDLINE contains the vast majority of studies published in the US, it is possible that articles indexed in other international databases were missed. Second, due to the expected heterogeneity of study types and the multiple outcomes included in the analyses, a qualitative appraisal of the potential sources of heterogeneity was conducted instead of a formal quality assessment using a standardized tool (such as the Cochrane Collaboration's tool for assessing risk of bias [63] or the Risk Of Bias In Non-randomised Studies-of Interventions [64]). Third, effect sizes and their variance directly extracted from publications and those calculated based on study results, as well as adjusted and unadjusted values, were included and treated similarly in the metaanalyses. Fourth, because of the relatively low number of studies included in the meta-analyses, sub-group analyses (e.g., based on study types, year of publication) or metaregressions could not be conducted to control for potentially confounding factors. Fifth, the presence of statistical heterogeneity between studies was confirmed by a high $I^{2}$ statistic in all analyses. While random effects meta-analyses aimed to account for heterogeneity between studies, differences may have remained in the studies' definitions of outcomes and patient populations. 


\section{Conclusion}

The findings of the present review suggest that LAI may significantly improve clinical outcomes for patients with schizophrenia. These include reduced hospitalizations and ER admissions, which are commonly used as proxy measures for disease relapses, and in turn are associated with substantial societal and economic burden. Further, the clinical benefits associated with LAI are achieved while remaining cost-neutral relative to $\mathrm{OA}$, through a reduction in medical costs offsetting pharmacy costs. Taken together with the finding of improved medication adherence, this may indicate better disease management among patients initiated on LAI versus OA. Given that the present analysis drew upon a wide range of data sources comprising patients enrolled in various healthcare plans, these clinical and economic outcomes are potentially generalizable to the broader population of patients with schizophrenia in the US and can support evidence-based decisions when considering LAI as a treatment option.

Supplementary Information The online version contains supplementary material available at https://doi.org/10.1007/s40263-021-00815-y.

Acknowledgements Medical writing assistance was provided by Mona Lisa Chanda, an employee of Analysis Group, Inc., which provided paid consulting services to Janssen Scientific Affairs, LLC to conduct this study.

\section{Declarations}

Funding This study was supported by Janssen Scientific Affairs, LLC. Open access funding was provided by Janssen Scientific Affairs, LLC.

Conflicts of interest DL, CB, and PM are employees of Janssen Scientific Affairs, LLC and may own stock/stock options. PT-L, IG, HN, M-HL, and PL are employees of Analysis Group, Inc., a consulting company that has provided paid consulting services to Janssen Scientific Affairs, LLC, to conduct this study.

Author contributions DL, PT-L, IG, HN, M-HL, CB, PM, and PL contributed to the design of the study and interpretation of the data. PT-L, IG, HN, and M-HL contributed to the data collection and data analysis. All authors critically revised the draft manuscript and approved the final content.

Availability of data and material Not applicable.

Code availability Not applicable.

Ethics approval Not applicable.

Consent to participate Not applicable.

Consent for publication Not applicable.
Open Access This article is licensed under a Creative Commons Attribution-NonCommercial 4.0 International License, which permits any non-commercial use, sharing, adaptation, distribution and reproduction in any medium or format, as long as you give appropriate credit to the original author(s) and the source, provide a link to the Creative Commons licence, and indicate if changes were made. The images or other third party material in this article are included in the article's Creative Commons licence, unless indicated otherwise in a credit line to the material. If material is not included in the article's Creative Commons licence and your intended use is not permitted by statutory regulation or exceeds the permitted use, you will need to obtain permission directly from the copyright holder. To view a copy of this licence, visit $\mathrm{http} / / /$ creativecommons.org/licenses/by-nc/4.0/.

\section{References}

1. Organization, W.H. Rolling updates on coronavirus disease (COVID-19). 2020; https://www.who.int/emergencies/diseases/ novel-coronavirus-2019/events-as-they-happen. Accessed 2 Sep 2020.

2. World Health Organization (WHO). Psychosis, including schizophrenia. 2019 [cited 201922 Oct]; http://www.emro.who.int/ health-topics/schizophrenia/index.html. Accessed 2 Sep 2020.

3. Emsley R, et al. The nature of relapse in schizophrenia. BMC Psychiatry. 2013;13:50.

4. Gopal S, et al. Caregiver burden in schizophrenia following paliperidone palmitate long acting injectables treatment: pooled analysis of two double-blind randomized phase three studies. NPJ Schizophr. 2017;3(1):23.

5. Lehman AF, et al. Practice guideline for the treatment of patients with schizophrenia, second edition. Am J Psychiatry. 2004;161(2 Suppl):1-56.

6. Wu EQ, et al. The economic burden of schizophrenia in the United States in 2002. J Clin Psychiatry. 2005;66(9):1122-9.

7. Cloutier M, et al. The economic burden of schizophrenia in the United States in 2013. J Clin Psychiatry. 2016;77(6):764-71.

8. Stevens GL, Dawson G, Zummo J. Clinical benefits and impact of early use of long-acting injectable antipsychotics for schizophrenia. Early Interv Psychiatry. 2016;10(5):365-77.

9. Keepers GA, et al. The American Psychiatric Association practice guideline for the treatment of patients with schizophrenia. Am J Psychiatry. 2020;177(9):868-72.

10. Correll, C.U., et al., A systematic literature review of schizophrenia clinical practice guidelines: recommendations on use of long-acting injectable antipsychotic medications. In: Presented at: Psych Congress 2020 Virtual Experience; Sep 10-13, 2020.

11. Weiden, P.J., et al., Efficacy and safety of a 2-month formulation of aripiprazole lauroxil with 1-day initiation in patients hospitalized for acute schizophrenia transitioned to outpatient care: phase 3, randomized, double-blind, active-control ALPINE Study. J Clin Psychiatry, 2020. 81(3):19m13207.

12. Parellada E, Bioque M. Barriers to the use of long-acting injectable antipsychotics in the management of schizophrenia. CNS Drugs. 2016; 30:689-701.

13. Kirschner M, et al. Patients' and clinicians' attitude towards long-acting depot antipsychotics in subjects with a first episode of psychosis. Ther Adv Psychopharmacol. 2013;3(2):89-99.

14. Carpenter WTJ, Buchanan RW. Expanding therapy with longacting antipsychotic medication in patients with schizophrenia. JAMA Psychiat. 2015;72(8):745-6.

15. Parellada E, et al. Long-acting injectable antipsychotics in first-episode schizophrenia. Schizophr Res Treatment. 2012;2012:318535. 
16. Bosanac P, Castle D. Why are long-acting injectable antipsychotics still underused? BJPscyh Adv. 2015;21(2):98-105.

17. Bera $\mathrm{R}$, et al. Impact on healthcare resource usage and costs among Medicaid-insured schizophrenia patients after initiation of treatment with long-acting injectable antipsychotics. J Med Econ. 2013;16(4):522-8.

18. Kishimoto $\mathrm{T}$, et al. Long-acting injectable versus oral antipsychotics in schizophrenia: a systematic review and meta-analysis of mirror-image studies. J Clin Psychiatry. 2013;74(10):957-65.

19. Kishimoto $\mathrm{T}$, et al. Indications for and use of long-acting injectable antipsychotics: consideration from an inpatient setting. Int Clin Psychopharmacol. 2017;32(3):161-8.

20. Offord $\mathrm{S}$, et al. Healthcare resource usage of schizophrenia patients initiating long-acting injectable antipsychotics vs oral. J Med Econ. 2013;16(2):231-9.

21. The University of South Florida; Florida Medicaid Drug Therapy Management Program sponsored by the Florida Agency for Health Care Administration. 2017-2018 Florida Best Practice Psychotherapeutic Medication Guidelines for Adults. 2018 [cited 20209 September]; https://medicaidmentalhealth.fmhi. usf.edu/_assets/file/Guidelines/2018-Psychotherapeutic\%20Med ication $\% 20$ Guidelines\%20for\%20Adults\%20with\%20Referenc es.pdf. Accessed 2 Sep 2020.

22. Pilon D, et al. Treatment patterns, health care resource utilization, and spending in medicaid beneficiaries initiating secondgeneration long-acting injectable agents versus oral atypical antipsychotics. Clin Ther. 2017;39(10):1972-1985.e2.

23. Marcus SC, et al. Antipsychotic adherence and rehospitalization in schizophrenia patients receiving oral versus long-acting injectable antipsychotics following hospital discharge. J Manag Care Spec Pharm. 2015;21(9):754-68.

24. Pilon D, et al. Adherence, healthcare resource utilization and Medicaid spending associated with once-monthly paliperidone palmitate versus oral atypical antipsychotic treatment among adults recently diagnosed with schizophrenia. BMC Psychiatry. 2017;17(1):207.

25. Baser $\mathrm{O}$, et al. Healthcare utilization and costs of Veterans Health Administration patients with schizophrenia treated with paliperidone palmitate long-acting injection or oral atypical antipsychotics. J Med Econ. 2015;18(5):357-65.

26. Alphs L, et al. A pragmatic analysis comparing once-monthly paliperidone palmitate versus daily oral antipsychotic treatment in patients with schizophrenia. Schizophr Res. 2016;170(2-3):259-64.

27. Alphs L, et al. Real-world outcomes of paliperidone palmitate compared to daily oral antipsychotic therapy in schizophrenia: a randomized, open-label, review board-blinded 15-month study. J Clin Psychiatry. 2015;76(5):554-61.

28. Lafeuille $\mathrm{MH}$, et al. Impact of atypical long-acting injectable versus oral antipsychotics on rehospitalization rates and emergency room visits among relapsed schizophrenia patients: a retrospective database analysis. BMC Psychiatry. 2013;13:221.

29. Pilon $\mathrm{D}$, et al. Short-term rehospitalizations in young adults with schizophrenia treated with once-monthly paliperidone palmitate or oral atypical antipsychotics: a retrospective analysis. Curr Med Res Opin. 2019;35(1):41-9.

30. Lafeuille $\mathrm{MH}$, et al. Comparison of rehospitalization rates and associated costs among patients with schizophrenia receiving paliperidone palmitate or oral antipsychotics. Am J Health Syst Pharm. 2015;72(5):378-89.

31. Burns T, Fiander M, Audini B. A delphi approach to characterising "relapse" as used in UK clinical practice. Int J Soc Psychiatry. 2000;46(3):220-30.

32. Lafeuille $\mathrm{MH}$, et al. Systematic review of long-acting injectables versus oral atypical antipsychotics on hospitalization in schizophrenia. Curr Med Res Opin. 2014;30(8):1643-55.
33. Olivares JM, et al. Definitions and drivers of relapse in patients with schizophrenia: a systematic literature review. Ann Gen Psychiatry. 2013;12(1):32.

34. Kishimoto T, et al. Effectiveness of long-acting injectable vs oral antipsychotics in patients with schizophrenia: a meta-analysis of prospective and retrospective cohort studies. Schizophr Bull. 2018;44(3):603-19.

35. Joshi K, et al. Real-world adherence and economic outcomes associated with paliperidone palmitate versus oral atypical antipsychotics in schizophrenia patients with substance-related disorders using Medicaid benefits. J Comp Eff Res. 2018;7(2):121-33.

36. Lefebvre $\mathrm{P}$, et al. Impact of paliperidone palmitate versus oral atypical antipsychotics on health care resource use and costs in veterans with schizophrenia and comorbid substance abuse. Clin Ther. 2017;39(7):1380-1395.e4.

37. Pesa JA, et al. Health care resource utilization and costs of California Medicaid patients with schizophrenia treated with paliperidone palmitate once monthly or atypical oral antipsychotic treatment. Curr Med Res Opin. 2017;33(4):723-31.

38. Pesa JA, et al. Costs and resource utilization among medicaid patients with schizophrenia treated with paliperidone palmitate or oral atypical antipsychotics. Drugs Real World Outcomes. 2015;2(4):377-85.

39. Young-Xu Y, et al. Impact of paliperidone palmitate versus oral atypical antipsychotics on health care resource use and costs in veterans with schizophrenia. J Clin Psychiatry. 2016;77(10):e1332-41.

40. Xiao Y, et al. Impact of paliperidone palmitate versus oral atypical antipsychotics on healthcare outcomes in schizophrenia patients. J Comp Eff Res. 2015;4(6):579-92.

41. Pilon D, et al. Adherence, persistence, and inpatient utilization among adult schizophrenia patients using once-monthly versus twice-monthly long-acting atypical antipsychotics. J Med Econ. 2018;21(2):135-43.

42. Rosenheck RA, et al. Cost-effectiveness of long-acting injectable paliperidone palmitate versus haloperidol decanoate in maintenance treatment of schizophrenia. Psychiatr Serv. 2016;67(10):1124-30.

43. Dekkers OM, et al. COSMOS-E: Guidance on conducting systematic reviews and meta-analyses of observational studies of etiology. PLoS Med. 2019;16(2):e1002742.

44. Tenny S, Hoffman MR. Odds Ratio (OR), in StatPearls. 2020. Treasure Island: StatPearls Publishing LLC.; 2020.

45. Rothman KJ, Greenland S, Lash TL. Modern epidemiology, 3rd edition, thoroughly revised and updated. Philadelphia: Wolters Kluwer Health/Lippincott Williams \& Wilkins; 2008.

46. Armitage P, Berry G, Matthews JNS. statistical methods in medical research. Chichester: John Wiley \& Sons; 2008.

47. Pilon D, et al. Treatment patterns in Medicaid patients with schizophrenia initiated on a first- or second-generation longacting injectable versus oral antipsychotic. Patient Prefer Adher. 2017;11:619-29.

48. Zhdanava, M., et al., Antipsychotic adherence, resource use, and costs before and after the initiation of once-monthly paliperidone palmitate therapy among medicaid beneficiaries with prior schizophrenia relapse. Clin Ther. 2021;S0149-2918(21)00015-1.

49. Patel, C., et al., Risk of subsequent relapses and corresponding healthcare costs among recently-relapsed Medicaid patients with schizophrenia: a real-world retrospective cohort study. Curr Med Res Opin. 2021;3(4):1-14.

50. Rozin E, et al. A retrospective study of the role of long-acting injectable antipsychotics in preventing rehospitalization in early psychosis with cannabis use. Addict Behav Rep. 2019;10:100221.

51. Heald, A., et al., Prescribing in schizophrenia and psychosis: Increasing polypharmacy over time. Hum Psychopharmacol, 2017. 32(2):e2579. 
52. Campagna EJ, et al. Methodological considerations in estimating adherence and persistence for a long-acting injectable medication. J Manag Care Spec Pharm. 2014;20(7):756-66.

53. Joshi K, et al. The Research and Evaluation of Antipsychotic Treatment in Community Behavioral Health Organizations, Outcomes (REACH-OUT) study: real-world clinical practice in schizophrenia. BMC Psychiatry. 2018;18(1):24.

54. Manjelievskaia J, et al. A comparison of treatment patterns, healthcare resource utilization, and costs among young adult Medicaid beneficiaries with schizophrenia treated with paliperidone palmitate or oral atypical antipsychotics in the US. J Med Econ. 2018;21(12):1221-9.

55. Shah A, et al. Treatment Patterns, healthcare resource utilization and costs among schizophrenia patients treated with long-acting injectable versus oral antipsychotics. Adv Ther. 2018;35(11):1994-2014.

56. San L, et al. Towards a pragmatic and operational definition of relapse in schizophrenia: a Delphi consensus approach. Int J Psychiatry Clin Pract. 2015;19(2):90-8.

57. Lin J, et al. Healthcare cost reductions associated with the use of LAI formulations of antipsychotic medications versus oral among patients with schizophrenia. J Behav Health Serv Res. 2013;40(3):355-66.

58. Peng $X$, et al. Decline in hospitalization risk and health care cost after initiation of depot antipsychotics in the treatment of schizophrenia. Clinicoecon Outcomes Res. 2011;3:9-14.

59. Masand PS, et al. Partial adherence to antipsychotic medication impacts the course of illness in patients with schizophrenia: a review. Prim Care Companion J Clin Psychiatry. 2009;11(4):147-54.

60. Kaplan G, Casoy J, Zummo J. Impact of long-acting injectable antipsychotics on medication adherence and clinical, functional, and economic outcomes of schizophrenia. Patient Prefer Adher. 2013;7:1171-80.

61. Haddad PM, Brain C, Scott J. Nonadherence with antipsychotic medication in schizophrenia: challenges and management strategies. Patient Relat Outcome Meas. 2014;5:43-62.

62. Sun SX, et al. Review and analysis of hospitalization costs associated with antipsychotic nonadherence in the treatment of schizophrenia in the United States. Curr Med Res Opin. 2007;23(10):2305-12.

63. Higgins JP, et al. The Cochrane Collaboration's tool for assessing risk of bias in randomised trials. BMJ. 2011;343:d5928.

64. Sterne JA, et al. ROBINS-I: a tool for assessing risk of bias in non-randomised studies of interventions. BMJ. 2016;355:i4919.

65. Lafeuille $\mathrm{MH}$, et al. Economic impact in medicaid beneficiaries with schizophrenia and cardiometabolic comorbidities treated with once-monthly Paliperidone palmitate vs. oral atypical antipsychotics. Drugs Real World Outcomes. 2018;5(1):81-90.

66. Greene $\mathrm{M}$, et al. Medication adherence and discontinuation of long-acting injectable versus oral antipsychotics in patients with schizophrenia or bipolar disorder. J Med Econ. 2017;21(2):127-34

67. Xiao Y, et al. Comparison of Medicaid spending in schizoaffective patients treated with once monthly paliperidone palmitate or oral atypical antipsychotics. Curr Med Res Opin. 2016;32(4):759-69.

68. Kamat, S.A., et al., Reduction in inpatient resource utilization and costs associated with long-acting injectable antipsychotics across different age groups of Medicaid-insured schizophrenia patients. Drugs Context. 2015;4:212267.

69. El Khoury A, et al. Transitioning from oral risperidone or paliperidone to once-monthly paliperidone palmitate: a real-world analysis among Veterans Health Administration patients with schizophrenia who have had at least one prior hospitalization. Curr Med Res Opin. 2019;35(12):2159-68.

70. Joshi K, et al. Adherence and economic impact of paliperidone palmitate versus oral atypical antipsychotics in a Medicare population. J Comp Eff Res. 2018;7(8):723-35.

71. Yan T, et al. Medication adherence and discontinuation of aripiprazole once-monthly $400 \mathrm{mg}$ (AOM 400) versus oral antipsychotics in patients with schizophrenia or bipolar I disorder: a real-world study using US Claims Data. Adv Ther. 2018;35(10):1612-25.

72. Anderson JP, et al. Comparison and predictors of treatment adherence and remission among patients with schizophrenia treated with paliperidone palmitate or atypical oral antipsychotics in community behavioral health organizations. BMC Psychiatry. 2017;17(1):346.

\section{Authors and Affiliations}

\section{Dee Lin ${ }^{1} \cdot$ Philippe Thompson-Leduc ${ }^{2} \cdot$ Isabelle Ghelerter ${ }^{2} \cdot$ Ha Nguyen $^{3} \cdot$ Marie-Hélène Lafeuille $^{2}$. Carmela Benson ${ }^{1} \cdot$ Panagiotis Mavros ${ }^{1} \cdot$ Patrick Lefebvre $^{2}$}

Philippe Thompson-Leduc

Philippe.Thompson-Leduc@analysisgroup.com

1 Janssen Scientific Affairs, LLC, Titusville, NJ, USA

2 Analysis Group, Inc., Montreal, QC, Canada
3 Analysis Group, Inc, Boston, MA, USA 